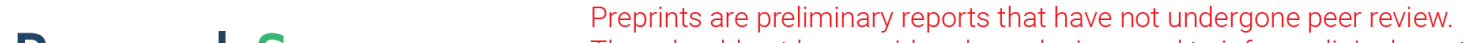 They should not be considered conclusive, used to inform clinical practice, or referenced by the media as validated information. \\ Breakdown of semiclassical description of thermoelectricity in near-magic angle twisted bilayer graphene
}

\section{Bhaskar Ghawri}

Indian Institute of Science Bangalore

Phanibhusan Mahapatra ( $\nabla$ phanis@iisc.ac.in )

Indian Institute of Science Bangalore

Manjari Garg

Indian Institute of Science Bangalore

Shinjan Mandal

Indian Institute of Science Bangalore https://orcid.org/0000-0003-1551-6555

Saisab Bhowmik

Indian Institute of Science

Aditya Jayraman

Indian Institute of Science Bangalore https://orcid.org/0000-0002-6963-7195

\section{Radhika Soni}

Indian Institute of Science

\section{Kenji Watanabe}

National Institute for Materials Science https://orcid.org/0000-0003-3701-8119

Takashi Taniguchi

National Institute for Materials Science, Tsukuba, Ibaraki https://orcid.org/0000-0002-1467-3105

Hulikal Krishnamurthy

Indian Institute of Science Bangalore

Manish Jain

Indian Institute of Science Bangalore

https://orcid.org/0000-0001-9329-6434

\section{Sumilan Banerjee}

Indian Institute of Science Bangalore

U. Chandni

Indian Institute of Science Bangalore

\section{Arindam Ghosh}

Indian Institute of Science Bangalore 
Keywords: twisted bilayer graphene (tBLG), non-Fermi liquid (NFL), van Hove singularities (vHS), SMR, hBN

Posted Date: August 26th, 2021

DOI: https://doi.org/10.21203/rs.3.rs-811957/v1

License: (c) (1) This work is licensed under a Creative Commons Attribution 4.0 International License. Read Full License

Version of Record: A version of this preprint was published at Nature Communications on March 21st, 2022. See the published version at https://doi.org/10.1038/s41467-022-29198-4. 


\title{
Breakdown of semiclassical description of thermoelectricity in near-magic angle twisted bilayer graphene
}

\author{
Bhaskar Ghawri, ${ }^{1,}$, Phanibhusan S. Mahapatra,, , Manjari Garg, ${ }^{2}$, 用 Shinjan Mandal, ${ }^{1}$ Saisab \\ Bhowmik, ${ }^{2}$ Aditya Jayaraman, ${ }^{1}$ Radhika Soni, ${ }^{2}$ Kenji Watanabe, ${ }^{3}$ Takashi Taniguchi, ${ }^{4}$ H. R. \\ Krishnamurthy, ${ }^{1}$ Manish Jain, ${ }^{1}$ Sumilan Banerjee, ${ }^{1}$ U. Chandni, ${ }^{2}$ and Arindam Ghosh ${ }^{1,5}, \$$ \\ ${ }^{1}$ Department of Physics, Indian Institute of Science, Bangalore, 560012, India \\ ${ }^{2}$ Department of Instrumentation and Applied Physics, \\ Indian Institute of Science, Bangalore, 560012, India \\ ${ }^{3}$ Research Center for Functional Materials, National Institute for \\ Materials Science, Namiki 1-1, Tsukuba, Ibaraki 305-0044, Japan \\ ${ }^{4}$ International Center for Materials Nanoarchitectonics, \\ National Institute for Materials Science, Namiki 1-1, Tsukuba, Ibaraki 305-0044, Japan \\ ${ }^{5}$ Centre for Nano Science and Engineering, Indian Institute of Science, Bangalore 560 012, India
}

\begin{abstract}
The planar assembly of twisted bilayer graphene (tBLG) hosts multitude of interaction-driven phases when the relative rotation is close to the magic angle $\left(\theta_{\mathrm{m}}=1.1^{\circ}\right)$. This includes correlationinduced ground states that reveal spontaneous symmetry breaking at low temperature, as well as possibility of non-Fermi liquid (NFL) excitations. However, experimentally, manifestation of NFL effects in transport properties of twisted bilayer graphene remains ambiguous. Here we report simultaneous measurements of electrical resistivity $(\rho)$ and thermoelectric power $(S)$ in tBLG for several twist angles between $\theta \sim 1.0-1.7^{\circ}$. We observe an emergent violation of the semiclassical Mott relation in the form of excess $S$ close to half-filling for $\theta \sim 1.6^{\circ}$ that vanishes for $\theta \gtrsim 2^{\circ}$. The excess $S\left(\approx 2 \mu \mathrm{V} / \mathrm{K}\right.$ at low temperatures $T \sim 10 \mathrm{~K}$ at $\left.\theta \approx 1.6^{\circ}\right)$ persists up to $\approx 40 \mathrm{~K}$, and is accompanied by metallic $T$-linear $\rho$ with transport scattering rate $\left(\tau^{-1}\right)$ of near-Planckian magnitude $\tau^{-1} \sim k_{\mathrm{B}} T / \hbar$. Closer to $\theta_{\mathrm{m}}$, the excess $S$ was also observed for fractional band filling $(\nu \approx 0.5)$. The combination of non-trivial electrical transport and violation of Mott relation provides compelling evidence of NFL physics intrinsic to tBLG.
\end{abstract}

In moiré systems with twisted bilayer graphene (tBLG), the amplification of Coulomb correlation effects at low twist angles $(\theta)$ is a result of nearly flat low-energy electronic bands [1, 2] and divergent density of states (DOS) at van Hove singularities (vHS) 3 . In addition to superconductivity [4], ferromagnetism [5], the strong correlation effects in tBLG manifest in a cascade of broken symmetry phases at integer band filling factor $(\nu)$ close to $\theta=\theta_{\mathrm{m}}$ [6, 7]. Near half-filling $(\nu= \pm 2)$ of the four-fold spin-valley degenerate conduction and valence bands, a linear $T$-dependence of the resistivity $(\rho)$ seems to indicate an interaction-related absence of a well-defined quasiparticle spectrum, which is concomitant with nonFermi liquid (NFL) excitations [8, 9]. The persistence of the linearity in $\rho$ for $\theta$ well away from $\theta_{\mathrm{m}}, e . g$. for $\theta \sim 1.5-2^{\circ}$, however, has been interpreted in terms of a contrary scenario that is addressable within the noninteracting framework 10 . The uncertainty persists even in scanning tunneling microscopy experiments [11 13, where the possibility of an interaction-driven magnetic order has been claimed close to the vHS for $\theta$ as high as $1.6^{\circ}$, although the spontaneous breaking of $C_{6}$ lattice symmetry to nematic orbital order has not been observed for $\theta>\theta_{\mathrm{m}}$. Thus a comprehensive understanding of the impact of correlation in tBLG requires a complementary experimental probe that is capable of identifying the departure from noninteracting physics in an unambiguous manner.

Here we have carried out simultaneous electrical and thermoelectric measurements in tBLG for twist angles varying from $\theta \sim 1.0-1.7^{\circ}$. The dependence on $T$ and on the carrier density $(n)$ of the thermoelectric power $(S)$, or the Seebeck coefficient, is used as an independent and sensitive probe of the correlation effects. Thermoelectric power is often interpreted as a thermodynamic entity that represents the entropy carried by each charge carrier. Within the degenerate quasiparticle description in the Boltzmann transport regime $\left(T \ll T_{\mathrm{F}}\right.$, where $T_{\mathrm{F}}$ is the Fermi temperature), $S$ is related to the resistance $(R)$ through the semiclassical Mott relation (SMR),

$$
S_{\mathrm{Mott}}=\left.\frac{\pi^{2} k_{\mathrm{B}}^{2} T}{3|e|} \frac{\mathrm{d} \ln R(E)}{\mathrm{d} E}\right|_{E_{\mathrm{F}}}
$$

where $R(E), e$ and $E_{\mathrm{F}}$ are the energy-dependent resistance, electronic charge and Fermi energy, respectively. Eq. 1 is valid under the assumption that scattering is elastic and isotropic close to the Fermi surface i.e. the transport lifetime only depends on the energy of the charge carriers. Remarkably, this simple assumption of isotropic scattering remains valid in a wide variety of systems, such as disordered metals/semiconductors [14, 15, organic materials [16, monolayer graphene [17] and topological insulators [18]. The SMR effectively arises from the quasiparticles carrying heat and charge under identical constraints, imposed by the momentum conservation. Thus, the validity of SMR in Eq. 1 provides a definitive probe into the nature of the scattering mechanisms and 

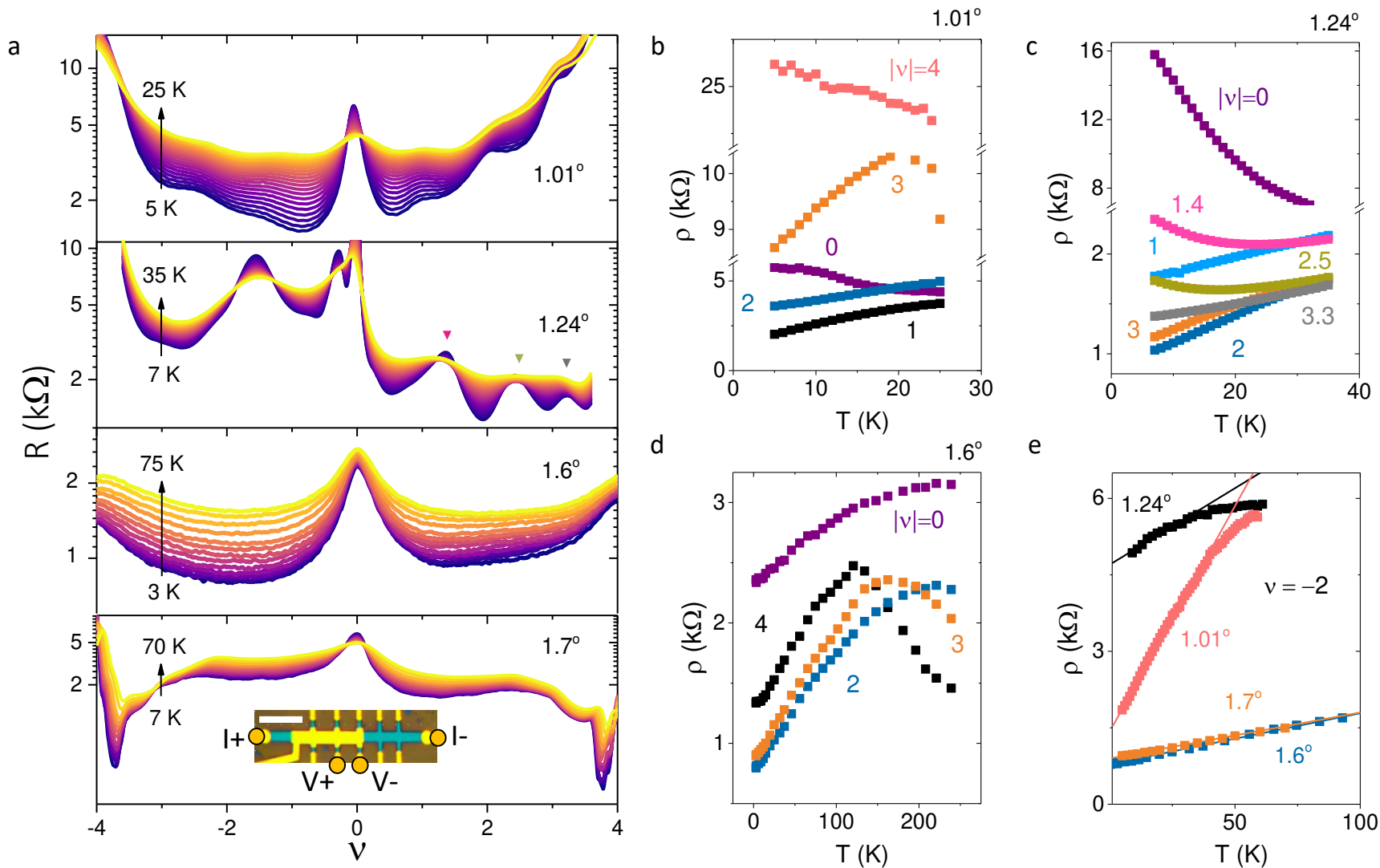

$\mathrm{e}$

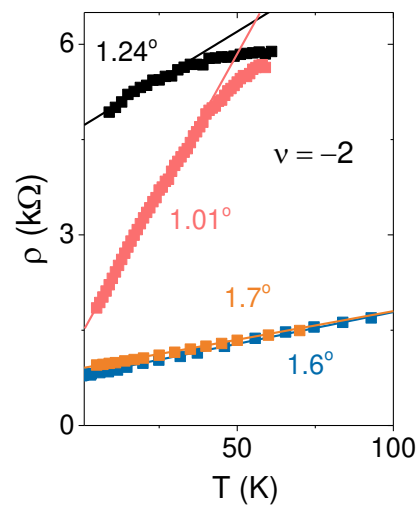

FIG. 1: Electrical transport in twisted bilayer graphene. (a) Temperature-dependent resistance $R$ as a function of band filling $\nu$ for four different devices with twist angle $\theta \approx 1.01^{\circ}, 1.24^{\circ}, 1.6^{\circ}$ and $1.7^{\circ}$. The inset in the bottom panel shows the optical image of a typical device with current and voltage leads marked for resistivity measurements. The scale bar represents a length of $5 \mu \mathrm{m} . \rho$ as a function of $T$ for a few representative values of $\nu$ for (b) $\theta \approx 1.01^{\circ}$, (c) $1.24^{\circ}$ and (d) $1.6^{\circ}$. The various curves for fractional $\nu$ in (c) represent the $T$-dependence of the CI/CS states as marked in the second panel of (a).(e) Comparison of $T$-dependence of $\rho$ at $\nu=-2$ for different twist angles. The solid lines represent $T$-linearity.

energy distribution of the charge carriers near the Fermi surface, and it breaks down when strong correlation effects become important [15, 19].

The tBLG devices we study were created using standard van der Waals stacking [20], which consists of two graphene layers aligned at either $60^{\circ}+\theta$ or at $\theta$, where $\theta$ is the effective twist angle, and encapsulated within two sheets of hexagonal boron nitride (hBN) (see supplementary information, SI, section I). A local top-gate tunes $n$ in the overlap region where the moiré super-lattice is formed. Fig. 1a shows the four terminal resistance $R$ measured across the tBLG devices as a function of band filling factor $\nu$ and $T$ for four different $\theta$. The recurring features in $R$ across the tBLG devices can be identified as the maxima in $R$ at CNP and at the full-filling of the moiré band $(\nu= \pm 4)$. In addition, near $\theta_{\mathrm{m}}$, the two devices $\theta \approx 1.01^{\circ}$ and $\approx 1.24^{\circ}$ exhibit additional maxima in $R$ near integer values of $\nu$, which can be mapped to the correlated states at integer fillings. For $\theta \approx 1.24^{\circ}$, we ob- serve a substantial shift $|\Delta \nu| \sim 0.25$ of resistance peaks near $\nu=+1$ and +3 from $7 \mathrm{~K}$ to $35 \mathrm{~K}$, suggesting the possibility of isospin-polarization in the system [21, 22] (SI, section III). We speculate that the noticeable asymmetry in the doping dependence of $R$ on the electron and hole sides is most likely related to the particle-hole asymmetry of the band structure since in both tBLG devices near $\theta_{\mathrm{m}}$, the correlated states are more pronounced at electron doping.

For $0<|\nu|<4$, the $T$-dependence for all devices was found to be generally metallic at low temperatures $\lesssim 40 \mathrm{~K}$ (Fig. 1b-1d). However, the resistance peaks near integer $\nu$ exhibit either weak insulating $T$-dependence, i.e, a correlated insulating phase $(\mathrm{CI})$, or $T$-linear resistivity i.e, a correlated semimetallic (CS) phase. In the metallic regime, $\rho$ can expressed as $\rho=\rho_{0}+A T$, where $\rho_{0}$ is the residual resistivity. The values of $A$ $(\sim 10-100 \Omega / \mathrm{K})$ are at least two orders of magnitude greater compared to that of the monolayer graphene, and 

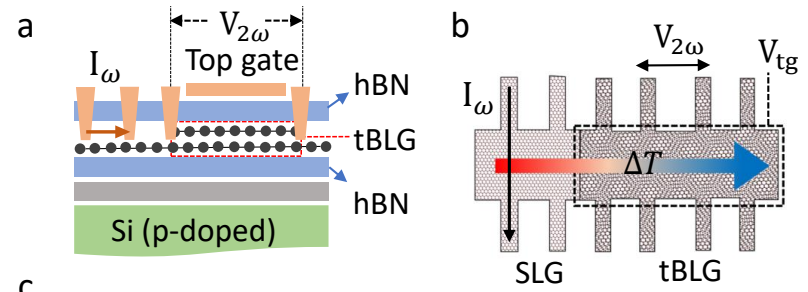

C

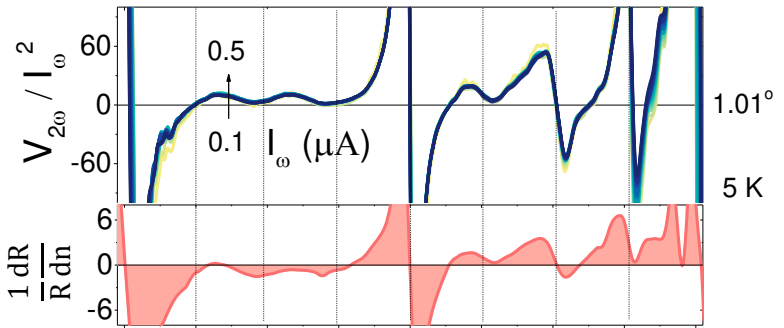

d

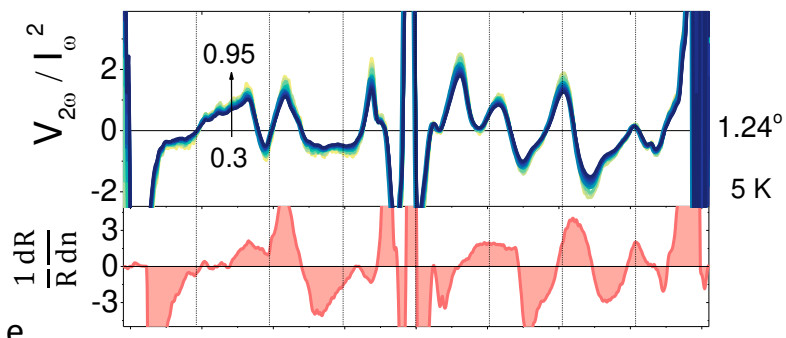

e

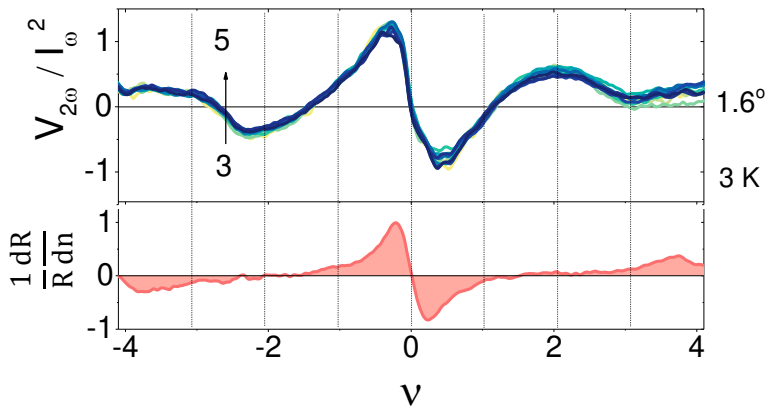

FIG. 2: Thermoelectric transport in twisted

bilayer graphene: (a) The cross-sectional view of the device, showing the constituent layers, electrical contacts, and the gate assembly. (b) In-plane heating and measurement schematic for thermovoltage $V_{2 \omega}$ in the tBLG region. Density dependence of $V_{2 \omega} / I_{\omega}^{2}$ (in the units of $\mathrm{VA}^{-2} \times 10^{6}$ ) measured at $5 \mathrm{~K}$ for (c) $\theta \approx 1.01^{\circ}$, (d) $1.24^{\circ}$, and at $3 \mathrm{~K}$ for (e) $1.6^{\circ}$ device. The different curves in each panel represent the $V_{2 \omega} / I_{\omega}^{2}$ measured at different $I_{\omega}$. The bottom graphs in each panel show the numerically calculated $\alpha=(1 / R) \mathrm{d} R / \mathrm{d} n$ (in the units of $\mathrm{m}^{2}$ ) for comparison.

are consistent with the earlier transport measurements in tBLG devices [10]. From the comparison of $\rho(T)$ at halffilling $(\nu=-2)$ in Fig. 1e, we find that the resistivity is linear in $T$ for all four low-angle devices. However, at $\theta \approx 1.24^{\circ}$ and $\theta \approx 1.01^{\circ}$, the linearity persists only upto $\sim 40 \mathrm{~K}$, which could be due to the smaller bandwidth and smaller bandgap [10]. We also note that the value of $A(\sim 40-100 \Omega / \mathrm{K})$ at $\nu=-2$ is much larger near $\theta \sim \theta_{\mathrm{m}}$ than that of the devices away from $\theta_{\mathrm{m}}$ (SI, section III). The ubiquitous $T$-linearity across all tBLG devices at low temperature is a clear departure from $\rho \sim T^{2}$ dependence associated with electron-electron scattering, or the $\rho \propto T^{4}$ behavior, expected due to electron-acoustic phonon scattering below the Bloch-Grüneisen temperature $\left(T_{\mathrm{BG}}\right)$ [23. While this suggests the continuum of correlation-driven metallic states across the tBLG devices, an alterate scenario has been proposed [10, 24] to view the tBLG in this regime as a two dimensional, weakly (or non-) interacting metal with largely reduced $T_{\mathrm{BG}}$.

To complement the electrical transport, we have performed thermoelectric measurements on the same devices. Briefly, a sinusoidal current $\left(I_{\omega}\right)$ is allowed to flow between two contacts of the monolayer branch outside the top gated region, setting up a temperature gradient $(\Delta T)$ across the tBLG region (Fig. 2a and 2b). The resulting second-harmonic thermo-voltage $\left(V_{2 \omega}\right)$ is recorded on the tBLG region as a function of doping and heating current [17, 20]. The linear response was ensured from $V_{2 \omega} \propto I_{\omega}^{2}$ for the range of heating current used (Fig. 2c-2e). We begin with the results in tBLG devices closer to $\theta_{\mathrm{m}}$. Fig. 2c exhibits the $\nu$-dependence of normalized $V_{2 \omega}$ for tBLG device $\theta \approx 1.01^{\circ}$ at low temperature $(5 \mathrm{~K})$, which exhibits multiple sign-reversals when $E_{\mathrm{F}}$ is varied across the lowest energy band. While the sign reversals near CNP and the super-lattice gaps at $\nu= \pm 4$ are due to changes in the quasiparticle excitations, those near integer values of $0<\nu<4$ can be attributed to the correlated states. The sign-reversal of $V_{2 \omega}$ near each correlated states is fascinating since it indicates a change in the topology of the Fermi surface, which is naturally associated with the Lifshitz transition [25, 26]. Although the correlated states are metallic in nature (Fig. 1a), the concomitant Lifshitz transitions depicts the interaction-driven occurrence of diverging DOS at each integer values of $\nu$. This is in stark contrast to the charge-inversions at $\nu=0, \pm 4$, and hints at the topological facet of the lowest energy band when filled with integer number of charge carriers [7, 27.

To establish the connection between the two different types of transports, we rewrite Eq. 1 as,

$$
S_{\mathrm{Mott}}=\left.\frac{\pi^{2} k_{\mathrm{B}}^{2} T}{3|e|} \frac{1}{R} \frac{\mathrm{d} R}{\mathrm{~d} V_{\mathrm{tg}}} \frac{\mathrm{d} V_{\mathrm{tg}}}{\mathrm{d} n} \frac{\mathrm{d} n}{\mathrm{~d} E}\right|_{E_{\mathrm{F}}},
$$

where $(1 / R) \mathrm{d} R / \mathrm{d} V_{\mathrm{tg}}$ is measured experimentally, and $\mathrm{d} n / \mathrm{d} E$ is the DOS $\left(\mathrm{d} V_{\mathrm{tg}} / \mathrm{d} n=e / C_{\mathrm{hBN}}\right.$, where $C_{\mathrm{hBN}}$ is the known topgate capacitance per unit area). The difficulty in accurate estimation of DOS in the presence of strong correlation effects near $\theta_{\mathrm{m}}$ prohibits us from accurately estimating $S_{\text {Mott }}$, in particular close to the 

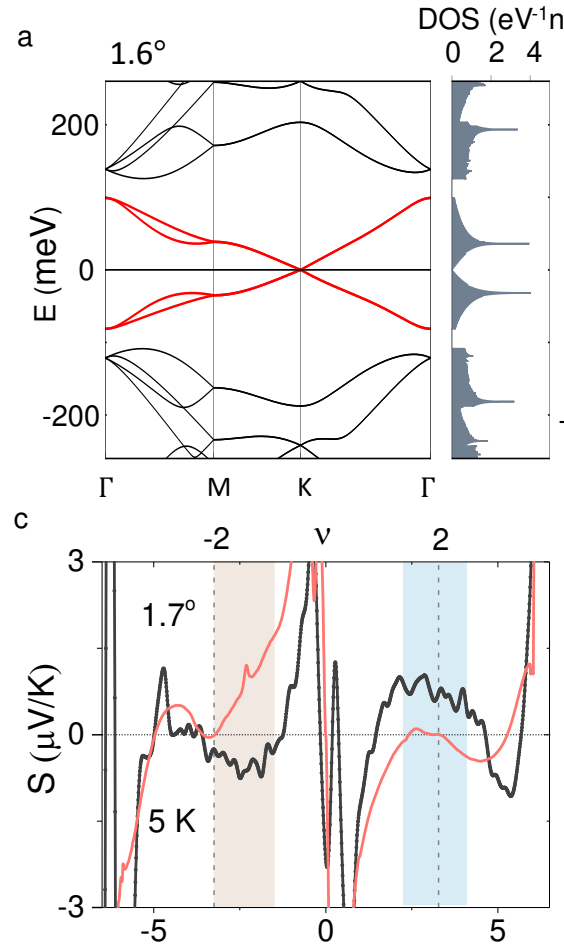

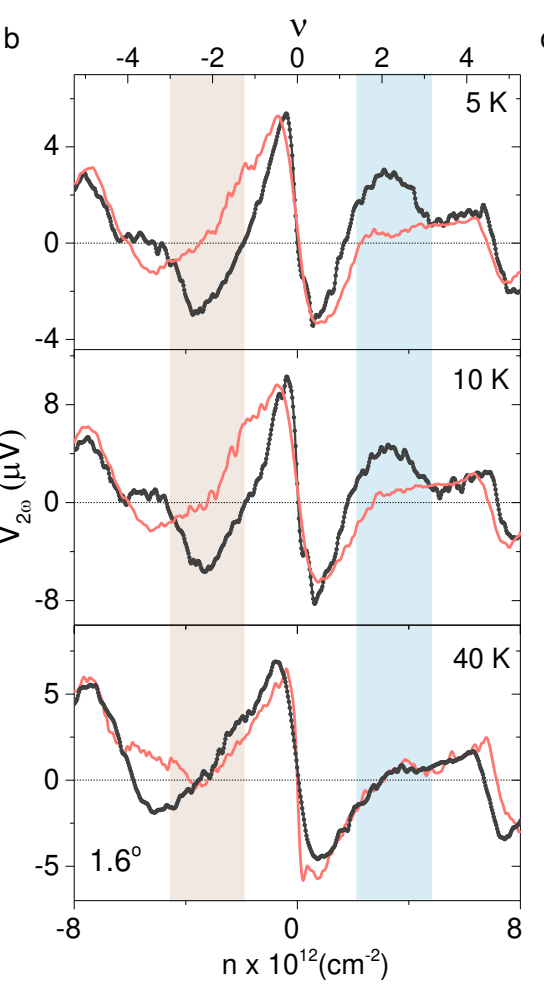

d

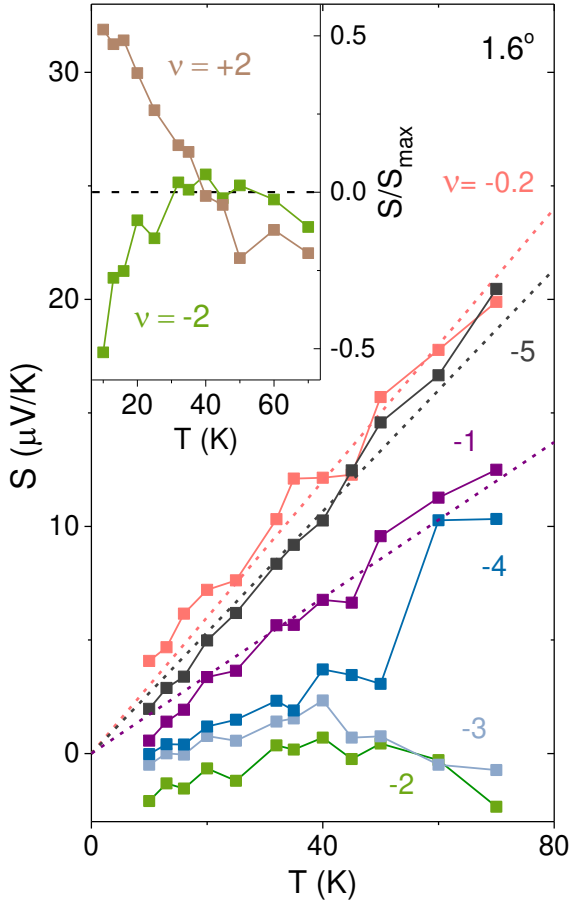

FIG. 3: Comparison with semiclassical Mott relation at $\sim 1.6^{\circ}$ (a) Electronic band structure and density of states (DOS) of tBLG $\left(\theta=1.6^{\circ}\right)$ calculated using tight binding model. The bands shown in red are the low energy active bands. (b) Comparison between the measured $V_{2 \omega}$ (black lines) and that calculated (orange line) from the semiclassical Mott relation (Eq. 2) for $\theta \approx 1.6^{\circ}$ at three representative temperatures. $\Delta T$ is obtained as a fitting parameter to match SMR with the experimental $V_{2 \omega}$ at CNP. (c) Doping dependence of $S$ for $\theta \approx 1.7^{\circ}$ compared to that of the SMR at $5 \mathrm{~K}$. (d) Temperature dependence of $S$ at various band filling factors. The dashed lines show the $S \propto T$ dependence. The inset shows the $T$ dependence of $S / S_{\max }$ at $\nu= \pm 2$.

integer fillings for $\theta \approx 1.01^{\circ}$ and $\approx 1.24^{\circ}$. Although a qualitative correspondence in the oscillations and signreversals of the measured $V_{2 \omega}$ and $\alpha=(1 / R) \mathrm{d} R / \mathrm{d} n$ can be seen in these devices including at the CNP and the superlattice gap, absence of accurate knowledge of the DOS prohibits a quantitative estimation of the deviation of the measured thermopower from that expected from the semiclassical model. However, for the device with $\theta \approx 1.24^{\circ}$ (Fig. $2 \mathrm{~d}$ ), we detect an excess $V_{2 \omega}$ near $\nu \sim 0.5$ which has no analogue in $\alpha$. While this indicates a clear violation of the Mott relation and highlights the possible manifestation of electron-correlation effects at fractional band filling [27, the exact origin of the excess $V_{2 \omega}$ at $\nu \approx 0.5$ is not clear at present.

Although the interaction-effects are expected to be weaker when $\theta$ is away from $\theta_{\mathrm{m}}$, the devices $\theta \approx 1.6^{\circ}$ and $1.7^{\circ}$ provide a better quantitative comparison with SMR as the non-interacting DOS can be calculated with greater accuracy. The qualitative comparison of $V_{2 \omega}$ with $\alpha$ at $\theta \approx 1.6^{\circ}$ exhibits a discrepancy at low temperature $(3 \mathrm{~K})$, where two additional extrema, consisting of a maximum at $\nu=+2$ and minimum at $\nu=-2$, are distinctly absent in $\alpha$ (Fig. 2e). Fig. 3a shows the tight binding calculation for the electronic band structure and the corresponding DOS for $\theta \approx 1.6^{\circ}$ (see Methods and SI, section VII for more details on the band structure calculations). Using $\Delta T$ as the single fitting parameter, we obtain excellent agreement between the measured $V_{2 \omega}$ and Eq. 2 at the CNP $(\nu=0), \nu= \pm 4$, and also in the higher energy dispersive band $(\nu> \pm 4)$ simultaneously (See SI, Fig. S16). We also note that $T \ll T_{\mathrm{F}}$ is maintained for fitting Eq. 2 at the CNP $(\nu=0)$ and $\nu= \pm 4$ throughout the temperature range shown in Fig. 3b (see SI, section V). While the SMR explains the observed $V_{2 \omega}$ over almost the entire doping regime $(-4 \lesssim \nu \lesssim+4)$ at high temperatures ( $\gtrsim 40 \mathrm{~K}$ ) (bottom panel of Fig. 3b), the excess thermovoltage centered around $\nu= \pm 2$, becomes evident at lower $T$. We also find that the excess thermovolatge is intrinsically particle-hole asymmetric, however, on the electron doped side, the excess thermovoltage is closer to the commensurate filling $(\nu=+2)$ as seen for two devices (Fig. 3b and 3c). We also detect ev- 

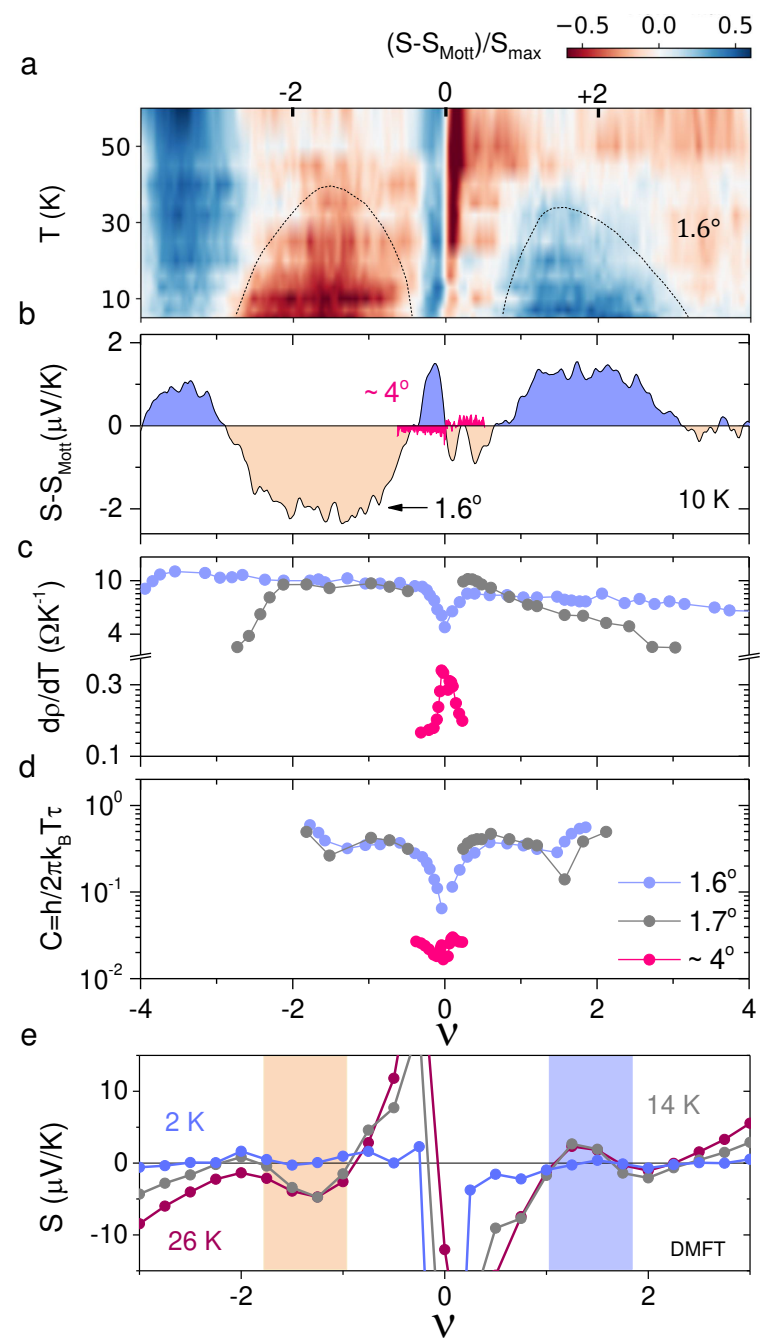

FIG. 4: Breakdown of semiclassical Mott relation and scattering rate : (a) Surface plot of $\left(S-S_{\text {Mott }}\right) / S_{\max }$ as a function of $T$ and $\nu$ for $\theta \approx 1.6^{\circ}$. (b) $\left(S-S_{\text {Mott }}\right)$ at $10 \mathrm{~K}$ for $\theta \approx 1.6^{\circ}$ and $\theta \sim 4^{\circ}$. (c) $\mathrm{d} \rho / \mathrm{d} T$ extracted in the $T$-linear regime at different $\nu$ for the three twist angles. (d) The estimated dimensionless pre-factor $C$ of the scattering rate $\Gamma=C k_{\mathrm{B}} T / \hbar$ as a function of $\nu$. (e) Seebeck coefficient $S$ computed in DMFT with $U=38 \mathrm{meV}$ as a function of filling $\nu$ for the four lowest bands at three temperatures $T=2 \mathrm{~K}, 14 \mathrm{~K}$ and $26 \mathrm{~K}$, respectively.

idence of small excess $V_{2 \omega}$ between $\nu=-3$ and -4 . This could also be due to electron-correlation effects, however, the exact origin is not clear as we do not observe any evidence of such anomalous thermopower near same filling factor in the other devices (see e.g. Fig. 3c for the device with $\theta \approx 1.7^{\circ}$ ). Using the $\Delta T$ extracted from the fitting of $V_{2 \omega}$, we show the $T$-dependence of $S=V_{2 \omega} / \Delta T$ in Fig. 3d for different $\nu$ (see SI, section V). Evidently, $S$ exhibits a linear dependence on $T$ at all doping including the higher-energy dispersive band, except in the vicinity of $\nu= \pm 2$, thus validating the estimation of $\Delta T$ from Mott fitting 25]. The $S \propto T$ behavior is expected in a degenerate weakly or non-interacting metal within the semiclassical framework, and has been verified for monolayer graphene [17] as well as tBLG at slightly larger $\theta$ $\left(2^{\circ} \lesssim \theta \lesssim 5^{\circ}\right)[28$. Close to $\nu= \pm 2$, however, we find an unexpected increase in $S$ when temperature is decreased below $\sim 40 \mathrm{~K}$, in contrast to the expectation of $S \approx 0$ (inset of Fig. 3d) from SMR and approaches $S \approx \pm 2 \mu \mathrm{V} / \mathrm{K}$ for $\nu= \pm 2$ respectively, at low $T$ (Fig. 3d and Fig. 4b). This is remarkable because, (1) at low $T$, the observed sign of $V_{2 \omega}$ can not be assigned to the electron(hole)like bands any more, and (2) the excess $S$ persists to a temperature scale $(\sim 40 \mathrm{~K})$ that is much higher than the superconducting transition $\left(T_{\mathrm{c}} \sim 1.7 \mathrm{~K}\right)$ in tBLG at $\theta=\theta_{\mathrm{m}}$ or the temperature scale for correlated insulator $(\lesssim 4 \mathrm{~K})$ [4, 6, 11, suggesting a very distinct nature of the ground state. The absolute magnitude of the excess thermovoltage at $\nu= \pm 2$ decreases with increasing $\theta$, as illustrated for a device with $\theta=1.7^{\circ}$ in Fig. 3c, and becomes undetectable for $\theta \gtrsim 2^{\circ}$.

Although the Mott formula has been verified in a range of graphene-based devices [17, 25], it can be violated in the hydrodynamic regime [29] and due to phonon drag in cross-plane thermoelectric transport in tBLG at $\theta>6^{\circ}$ [20. While the hydrodynamic regime is expected to appear at higher temperatures $(>100 \mathrm{~K})$, we eliminate the possibility of phonon drag from the observation of $S \propto T$ (away from $\nu= \pm 2$, Fig. 3d). Furthermore, as shown in Fig. 4a, the occurrence of excess $S$, normalized as $\left(S-S_{\text {Mott }}\right) / S_{\max }$, where $S_{\max }$ is the maximum value of $S$ at a given $T$, is concentrated in the low $T$ dome-like regions around $\nu= \pm 2$ in the $T-(\nu, n)$ phase diagram. Since neither adiabatic (static) nor dynamical phonon effects can violate Mott formula [30, 31, the enhancement of thermopower beyond the SMR limit suggests the possibility of a many body ground state similar to NFL phases in correlated oxides 32 and heavy Fermions [33. A near-ubiquitous feature of the NFL regime in itinerant Fermionic systems, ranging from cuprates [34, ruthanates [35, pnictides [36] to heavy Fermions [33], is the 'strange metal' phase, characterized by the absence of well defined quasiparticles and linear $T$ dependence of $\rho$. Theoretical work also suggests possibilities of excess entropy, analogous to Bekenstein-Hawking entropy in charged black holes, in this regime, that remains finite down to vanishingly small $T$ 37.

To check the mutuality between the excess thermopower and the strange metallic behaviour, we compare the $\nu$-dependence of excess $S$ at $T=10 \mathrm{~K}$ (Fig. 4b), and the scattering rate obtained from the slope $\mathrm{d} \rho / \mathrm{d} T$ in the $T$-dependence of $\rho$ (Fig. 4c). For reference, we also present the results from another device at $\theta \approx 4^{\circ}$, where we find no violation of SMR over the experimental range of $n$. In the NFL state, the incoherent scattering rate is 
$\tau^{-1}=C k_{\mathrm{B}} T / \hbar$, where the dimensionless coefficient $C$ is of the order of unity for Planckian dissipation. In Fig. $4 \mathrm{c}$ we plot the $\nu$-dependence of $\mathrm{d} \rho / \mathrm{d} T$ and $C$ (Fig. $4 \mathrm{~d}$ ), where $C$ is computed from $\mathrm{d} \rho / \mathrm{d} T$ assuming Drude-like resistivity in accordance to Ref. [8, 9] (See SI, section $\mathrm{VI}$ ). Away from the CNP, both $1.6^{\circ}$ and $1.7^{\circ}$ devices show $\mathrm{d} \rho / \mathrm{d} T \approx 10 \Omega / \mathrm{K}$ near $\nu \approx \pm 2$, which is nearly two orders of magnitude larger than $\mathrm{d} \rho / \mathrm{d} T \approx 0.2-0.3 \Omega / \mathrm{K}$ for the tBLG device at $\theta \sim 4^{\circ}$, implying that the individual layers are essentially decoupled in the latter [8, 10. Intriguingly, for tBLG at $\theta=1.6^{\circ}$ and $1.7^{\circ}$, we find $C$ to approach the order of unity in the vicinity of $\nu \rightarrow \pm 2$, raising the possibility of a common physical origin for the violation of SMR. Notably, the excess thermopower was found largely unaffected in the in-plane magnetic field (Fig. S20, SI, section V), and thus unlikely to arise from an underlying spin/magnetic texture [5. Theoretically a dynamical mean field theory (DMFT) 33, 38, 39] calculation shows qualitative agreement in the density dependence of excess thermopower at $\nu= \pm 2$ but fails to capture its finite magnitude at low temperature (Fig. 4e). This is because the particular single site DMFT framework used in our calculation would invariably lead to the FL phase as $T \rightarrow 0$, even though some excess thermopower can be observed in the intermediate temperature range (see SI, sections VII for details).

In summary, we have measured the electrical resistivity and thermopower in twisted bilayer graphene over a broad range of low-twist angles. At larger $\theta\left(\sim 1.6^{\circ}-\right.$ $1.7^{\circ}$ ), our experimental results show concurrent $T$-linear resistivity at Planckian dissipation scales and emergent excess thermopower below $T \lesssim 40 \mathrm{~K}$ near $\nu= \pm 2$ signifying the breakdown of the semiclassical Mott relation. The thermopower near $\nu= \pm 2$ approaches a finite magnitude $\left(\approx 2 \mu \mathrm{V} / \mathrm{K}\right.$ at $\left.1.6^{\circ}\right)$ at low $T$ providing a new facet to the strongly correlated 'strange metal' phase in tBLG. Our experimental results point to a truly nonFermi liquid (NFL) metallic state in tBLG at low twist angle that carry strong similarities to those observed in cuprates or heavy-Fermion materials with low coherence temperatures.

The authors thank Nano mission, DST for the financial support. M.J. and S.M. thank the computational facilities in SERC. K.W. and T.T. acknowledge support from the Elemental Strategy Initiative conducted by the MEXT, Japan, Grant Number JPMXP0112101001, JSPS KAKENHI Grant Numbers JP20H00354 and the CREST(JPMJCR15F3), JST. U.C. acknowledges funding from IISc and SERB (ECR/2017/001566), and H.R.K from SERB(SB/DF/005/2017). S.B. acknowledges funding from IISc and SERB (ECR/2018/001742).

B.G., P.S.M. and MG contributed equally to this work.

\section{METHODS}

\section{Device fabrication}

All devices in this work were fabricated using a layerby-layer mechanical transfer method 20]. Monolayer graphene and hexagonal boron nitride $(\mathrm{hBN})$ were exfoliated on $\mathrm{SiO}_{2} / \mathrm{Si}$ wafers and graphene flakes were identified using optical microscopy and Raman spectroscopy. For $\theta \approx 1.6^{\circ}$, the edges of the graphene flakes were aligned under an optical microscope and encapsulated within two hBN layers. Other tBLG devices were fabricated using tear and stack method [40. Electron beam lithography was used to define $\mathrm{Cr} / \mathrm{Au}$ top gate for tuning the number density in the tBLG region. Finally, the electrical contacts were patterned by electron-beam lithography and reactive ion etching followed by metal deposition (5 $\mathrm{nm} \mathrm{Cr} / 50 \mathrm{~nm} \mathrm{Au}$ ) using thermal evaporation technique.

Electrical transport measurements were performed in a four-terminal geometry with typical $a c$ current excitations of 10-100 nA using a standard low-frequency lock-in amplifier at $226 \mathrm{~Hz}$, in a dilution refrigerator and a $1.5-\mathrm{K}$ cryostat. For thermoelectric measurements, local Joule heating was employed to create a $\Delta T$ across the tBLG channel. A range of sinusoidal currents $(2-5 \mu \mathrm{A})$ at excitation frequency $\omega=17 \mathrm{~Hz}$ were used for Joule heating and the resulting $2^{\text {nd }}$ harmonic thermal voltage $\left(V_{2 \omega}\right)$ was recorded using a lock-in amplifier. Thermoelectric measurements were conducted in a $1.5-\mathrm{K}$ cryostat $/ 20 \mathrm{mK}$ dilution refrigerator with magnetic field.

\section{Tight binding calculation of DOS}

The rigid bilayer structures were generated using the Twister code [41. The structures were subsequently relaxed in LAMMPS [42, 43] using REBO [44] as the intralayer potential and DRIP [45] as the interlayer potential. These relaxed structures were used for performing all the calculations.

The electronic band structures were calculated by approximating the tight binding transfer integrals under the Slater Koster formalism [46]. A more detailed discussion of the calculations is available in the SI, section VII.

\footnotetext{
* gbhaskar@iisc.ac.in

$\dagger$ phanis@iisc.ac.in

¥ manjarigarg@iisc.ac.in

$\S$ arindam@iisc.ac.in

[1] Trambly de Laissardière, G., Mayou, D. \& Magaud, L. Localization of Dirac electrons in rotated graphene bilayers. Nano Lett. 10, 804-808 (2010).
} 
[2] Bistritzer, R. \& MacDonald, A. H. Moiré bands in twisted double-layer graphene. Proc. Natl Acad. Sci. 108, 12233-12237 (2011).

[3] Yuan, N. F. Q., Isobe, H. \& Fu, L. Magic of high-order van Hove singularity. Nat. Comm. 10 (2019).

[4] Cao, Y. et al. Unconventional superconductivity in magic-angle graphene superlattices. Nature 556, 43 (2018).

[5] Sharpe, A. L. et al. Emergent ferromagnetism near threequarters filling in twisted bilayer graphene. Science $\mathbf{3 6 5}$, 605-608 (2019).

[6] Cao, Y. et al. Correlated insulator behaviour at halffilling in magic-angle graphene superlattices. Nature 556, 80 (2018).

[7] Zondiner, U. et al. Cascade of phase transitions and dirac revivals in magic-angle graphene. Nature 582, 203-208 (2020).

[8] Cao, Y. et al. Strange metal in magic-angle graphene with near Planckian dissipation. Phys. Rev. Lett. 124, 076801 (2020).

[9] Bruin, J., Sakai, H., Perry, R. \& Mackenzie, A. Similarity of scattering rates in metals showing T-linear resistivity. Science 339, 804-807 (2013).

[10] Polshyn, H. et al. Large linear-in-temperature resistivity in twisted bilayer graphene. Nat. Phys. 15, 1011-1016 (2019).

[11] Kerelsky, A. et al. Maximized electron interactions at the magic angle in twisted bilayer graphene. Nature 572, 95100 (2019).

[12] Jiang, Y. et al. Charge order and broken rotational symmetry in magic-angle twisted bilayer graphene. Nature 573, 91-95 (2019).

[13] Liu, Y.-W. et al. Magnetism near half-filling of a van hove singularity in twisted graphene bilayer. Phys. Rev. B 99, 201408 (2019).

[14] Rowe, D. M. Materials, preparation, and characterization in thermoelectrics (CRC press, 2017).

[15] Behnia, K. Fundamentals of thermoelectricity (OUP Oxford, 2015).

[16] Watanabe, S. et al. Validity of the Mott formula and the origin of thermopower in $\pi$-conjugated semicrystalline polymers. Phys. Rev. B 100, 241201 (2019).

[17] Zuev, Y. M., Chang, W. \& Kim, P. Thermoelectric and magnetothermoelectric transport measurements of graphene. Phys. Rev. Lett. 102, 096807 (2009).

[18] Kim, D., Syers, P., Butch, N. P., Paglione, J. \& Fuhrer, M. S. Ambipolar surface state thermoelectric power of topological insulator $\mathrm{Bi}_{2} \mathrm{Se}_{3}$. Nano Lett. 14, 1701-1706 (2014).

[19] Arsenijević, S. et al. Signatures of quantum criticality in the thermopower of $\mathrm{Ba}\left(\mathrm{Fe}_{1-x} \mathrm{Co}_{x}\right)_{2} \mathrm{As}_{2}$. Phys. Rev. B 87, 224508 (2013).

[20] Mahapatra, P. S., Sarkar, K., Krishnamurthy, H. R., Mukerjee, S. \& Ghosh, A. Seebeck coefficient of a single van der Waals junction in twisted bilayer graphene. Nano Lett. 17, 6822-6827 (2017).

[21] Saito, Y. et al. Isospin pomeranchuk effect and the entropy of collective excitations in twisted bilayer graphene. arXiv preprint arXiv:2008.10830 (2020).

[22] Rozen, A. et al. Entropic evidence for a pomeranchuk effect in magic angle graphene. arXiv preprint arXiv:2009.01836 (2020).

[23] Efetov, D. K. \& Kim, P. Controlling electron-phonon interactions in graphene at ultrahigh carrier densities.
Phys. Rev. Lett. 105, 256805 (2010).

[24] Wu, F., Hwang, E. \& Sarma, S. D. Phonon-induced giant linear-in- $\mathrm{T}$ resistivity in magic angle twisted bilayer graphene: Ordinary strangeness and exotic superconductivity. Phys. Rev. B 99, 165112 (2019).

[25] Jayaraman, A. et al. Evidence of lifshitz transition in the thermoelectric power of ultrahigh-mobility bilayer graphene. Nano Lett. 21, 1221-1227 (2021).

[26] Buhmann, J. M. \& Sigrist, M. Thermoelectric effect of correlated metals: Band-structure effects and the breakdown of Mott's formula. Phys. Rev. B 88, 115128 (2013).

[27] Wu, S., Zhang, Z., Watanabe, K., Taniguchi, T. \& Andrei, E. Y. Chern insulators, van hove singularities and topological flat bands in magic-angle twisted bilayer graphene. Nat. Mat. 20, 488-494 (2021).

[28] Mahapatra, P. S. et al. Misorientation-controlled crossplane thermoelectricity in twisted bilayer graphene. Phys. Rev. Lett. 125, 226802 (2020).

[29] Ghahari, F. et al. Enhanced thermoelectric power in graphene: Violation of the Mott relation by inelastic scattering. Phys. Rev. Lett. 116, 136802 (2016).

[30] Jonson, M. \& Mahan, G. Mott's formula for the thermopower and the Wiedemann-Franz law. Phys. Rev. B 21, 4223 (1980).

[31] Jonson, M. \& Mahan, G. Electron-phonon contribution to the thermopower of metals. Phys. Rev. B 42, 9350 (1990).

[32] Wang, Y., Rogado, N. S., Cava, R. J. \& Ong, N. P. Spin entropy as the likely source of enhanced thermopower in $\mathrm{Na}_{x} \mathrm{Co}_{2} \mathrm{O}_{4}$. Nature 423, 425-428 (2003).

[33] Izawa, K. et al. Thermoelectric response near a quantum critical point: The case of $\mathrm{CeCoIn}_{5}$. Phys. Rev. Lett. 99, 147005 (2007).

[34] da Silva Neto, E. H. et al. Ubiquitous interplay between charge ordering and high-temperature superconductivity in cuprates. Science 343, 393-396 (2014).

[35] Rost, A., Perry, R., Mercure, J.-F., Mackenzie, A. \& Grigera, S. Entropy landscape of phase formation associated with quantum criticality in $\mathrm{Sr}_{3} \mathrm{Ru}_{2} \mathrm{O}_{7}$. Science 325, 1360-1363 (2009).

[36] Lee, W.-C. \& Phillips, P. W. Non-Fermi liquid due to orbital fluctuations in iron pnictide superconductors. Phys. Rev. B 86, 245113 (2012).

[37] Sachdev, S. Bekenstein-Hawking entropy and strange metals. Phys. Rev. X 5, 041025 (2015).

[38] Georges, A., Kotliar, G., Krauth, W. \& Rozenberg, M. J. Dynamical mean-field theory of strongly correlated fermion systems and the limit of infinite dimensions. Rev. Mod. Phys. 68, 13-125 (1996).

[39] Haldar, A., Banerjee, S. \& Shenoy, V. B. Higherdimensional Sachdev-Ye-Kitaev non-Fermi liquids at Lifshitz transitions. Phys. Rev. B 97, 241106 (2018).

[40] Kim, K. et al. Tunable moiré bands and strong correlations in small-twist-angle bilayer graphene. Proceedings of the National Academy of Sciences 114, 3364-3369 (2017).

[41] Naik, M. H. \& Jain, M. Ultraflatbands and shear solitons in moire patterns of twisted bilayer transition metal dichalcogenides. Phys. Rev. Lett. 121, 266401 (2018).

[42] Plimpton, S. Fast parallel algorithms for short-range molecular dynamics (1993).

[43] https://lammps.sandia.gov .

[44] Brenner, D. W. et al. A second-generation reactive empirical bond order (rebo) potential energy expression for 
hydrocarbons. J Phys. Cond. Mat. 14, 783 (2002).

[45] Wen, M., Carr, S., Fang, S., Kaxiras, E. \& Tadmor, E. B. Dihedral-angle-corrected registry-dependent interlayer potential for multilayer graphene structures. Phys.
Rev. B 98, 235404 (2018).

[46] Slater, J. C. \& Koster, G. F. Simplified LCAO method for the periodic potential problem. Phys. Rev. 94, 1498 (1954). 


\section{Supplementary Files}

This is a list of supplementary files associated with this preprint. Click to download.

- Supplementary.pdf 